\title{
PLANILHA ELETRÔNICA PARA ESTIMATIVAS DE FUGACIDADES PARCIAIS USANDO EQUAÇÃO DO VIRIAL/HAYDEN-O'CONNELL
}

\author{
J. S. da SILVA NETO ${ }^{1}$, P. H. M da SILVA ${ }^{1}$, T. O. B. FONSECA, J. A. CAVALCANTE ${ }^{1}$ e N. A. $\operatorname{COSTA}^{1}$ \\ ${ }^{1}$ Universidade Federal da Paraíba \\ E-mail: nagelalves@hotmail.com
}

\begin{abstract}
RESUMO - O trabalho tem por objetivo fornecer uma planilha eletrônica para as estimativas de fugacidades de espécies puras, de misturas e de espécies $i$ presentes na mistura através da equação do virial com o segundo coeficiente estimado através da correlação de HaydenO'Connell. O trabalho é dividido em cinco partes: a) criação do banco de dados contendo: propriedades críticas e parâmetros moleculares; b) criação de caixas de diálogos para interação do usuário com o programa, c) codificações de subprogramas VBA para as estimativas dos coeficientes do virial das espécies puras e cruzadas, d) codificações de subprogramas VBA para avaliações das propriedades volumétricas e fugacidades usando a equação do virial das expansões na pressão e na densidade molar e e) validação dos resultados fornecidos pelo programa. O resultado final é um aplicativo para fins educacionais e/ou comerciais, capaz de fornecer estimativas das propriedades volumétricas e fugacidades com rapidez e confiabilidade.
\end{abstract}

\section{INTRODUÇÃO}

No método preditivo proposto por Hayden e O'Connell (1975), o segundo coeficiente do virial é influenciado através dos vários tipos de forças que atuam nas moléculas. Os pesquisadores expressaram o segundo coeficiente do virial como à soma de duas contribuições individuais: a) forças físicas (forças fracas - volumétricas ou de van der Waals); b) forças químicas (forças fortes - ligação de hidrogênio e dimerização das moléculas). O resumo é apresentado no Quadro 1. A modelagem matemática é descrita em Costa (2013a, 2013b).

Quadro 1 - Resumo da correlação de Hayden-O'Connel

Segundo coeficiente: $B_{i j}=B_{i j}^{F}+B_{i j}^{D}$

Segundo coeficiente - forças volumétricas: $B_{i j}^{F}=\left(B_{\text {apolar }}^{F}\right)_{i j}+\left(B_{\text {polar }}^{F}\right)_{i j}$

Segundo coeficiente - interações apolares: $\left(B_{\text {apolar }}^{F}\right)_{i j}=b_{0 i j}\left(0,94-1,47 T_{i j}^{*}-0,85 T_{i j}^{* 2}+1,015 T_{i j}^{* 3}\right)$

Segundo coeficiente - interações polares: $\left(B_{\text {polar }}^{F}\right)_{i j}=-b_{0 i j} \mu_{i j}^{*}\left(0,75-3,0 T_{i j}^{*}+2,1 T_{i j}^{* 2}+2,1 T_{i j}^{* 3}\right)$

Segundo coeficiente - forças químicas: $B_{i j}^{D}=\left(B_{\text {metastable }}+B_{\text {bound }}\right)_{i j}+\left(B_{\text {chemical }}\right)_{i j}$ 
Segundo coeficiente - efeito das ligações: $\left(B_{\text {metastable }}+B_{\text {bound }}\right)_{i j}=b_{0 i j} A_{i j} \exp \left(\frac{\Delta H_{i j}}{T_{r i j}}\right)$

Segundo coeficiente - associações moleculares: $\left(B_{\text {Chemical }}\right)_{i j}=b_{0 i j} E_{i j}\left[1-\exp \left(\frac{1500 \eta_{i j}}{T}\right)\right]$

Para misturas multicomponentes, o procedimento para a estimativa do coeficiente do virial pode ser dividido em duas partes: a) avaliação do segundo coeficiente do virial de cada componente puro presente na mistura. As informações necessárias são as propriedades críticas $\left(T_{c}\right.$ e $\left.P_{c}\right)$ e raio médio de giração, $\bar{R}_{i}$, momento dipolo $\mu_{i i}$ e parâmetro de associação $\eta_{i i}$; e b) avaliação do segundo coeficiente do virial cruzado de cada par de componentes presentes na mistura usando as regras de mistura proposta por Hayden e O'Connell (1975). As informações necessárias são os parâmetros dos componentes puros: $(\varepsilon / k)_{i i}, \sigma_{i i}$ e $w_{i i}$ para i $=1,2, \ldots, N$ e parâmetros de solvatação. As correlações empregadas nas estimativas dos vários tipos de coeficientes descritos no Quadro 1 são apresentadas no Quadro 2.

Quadro 2 - Parâmetros da correlação de Hayden-O'Connell

Temperatura reduzida e pseudo-reduzida: $T_{r i j}=\frac{T}{(\varepsilon / k)_{i j}}$ e $T_{i j}^{*}=\frac{1}{T_{r i j}}-1,6 w_{i j}$

- Fator acêntrico: $w_{i i}=0,006 \bar{R}_{i}+0,02087 \bar{R}_{i}^{2}-0,00136 \bar{R}_{i}^{3}$ e $w_{i j}=\frac{w_{i i}+w_{i j}}{2}$

Energia intermolecular sem correção de polaridade: $(\varepsilon / k)_{i}=T_{c i}\left[0,748+0,91 w_{i}-\frac{0,4 \eta_{i}}{\left(2+20 w_{i}\right)}\right]$

Parâmetro de tamanho molecular sem correção de polaridade $\sigma_{i}^{3}=\left(2,44-w_{i}\right)^{3}\left(\frac{T_{c i}}{P_{c i}}\right)$

Volume molar característico em cm³/mol: $b_{0 i j}=1,26184 \sigma_{i j}^{3}$

Momento dipolo pseudo-reduzido: $\mu_{i j}^{*}= \begin{cases}\mu_{r i j} & \text { Para } 0 \leq \mu_{r i j}<0,04 \\ 0 & \text { Para } 0,04 \leq \mu_{r i j}<0,25 \\ \mu_{r i j}-0,25 & \text { Para } \mu_{r i j} \geq 0,25\end{cases}$

Parâmetro $A: A_{i j}=-0,3-0,05 \mu_{r i j}$

Momento dipolo reduzido, $\mu_{r i}: \mu_{r i j}=\frac{7243,8 \mu_{i} \mu_{j}}{(\varepsilon / k)_{i j} \sigma_{i j}^{3}}$

Entalpia de formação efetiva: $\Delta H_{i j}=1,99+0,2 \mu_{r i j}^{2}$ 
Parâmetro $E: E_{i j}= \begin{cases}\exp \left[\eta_{i j}\left(\frac{650}{(\varepsilon / k)_{i j}+300}-4,27\right)\right] & \text { Para } \eta_{i j}<4,5 \\ \exp \left[\eta_{i j}\left(\frac{42800}{(\varepsilon / k)_{i j}+22400}-4,27\right)\right] & \text { Para } \eta_{i j} \geq 4,5\end{cases}$

Na ausência de informações experimentais para os parâmetros de solvatação, Fredenslund et al. (1977) fornecem roteiros, descritos nas Tabelas 1 e 2, para as estimativas desses parâmetros.

Tabela 1 - Parâmetros de solvatação

\begin{tabular}{l|l}
\hline Misturas contendo: & $\eta_{i j}$ \\
\hline Componentes apolares & 0 \\
Hidrocarbonetos & 0 \\
Hidrocarbonetos e componentes apolares (exceto fluorocarbonos) & 0 \\
Hidrocarbonetos alifáticos e um componente polar & 0 \\
\hline
\end{tabular}

Tabela 2 - Parâmetros de solvatação para compostos orgânicos e água

\begin{tabular}{ll|cccccc}
\hline & 1 & 2 & 3 & 4 & 5 & 6 \\
\hline 1 & Hidrocarbonetos aromáticos & 0 & 0 & 0 & 0,5 & 0,6 & 0,4 \\
2 & Alcoóis & & 1,55 & 1,55 & 1,0 & 1,3 & 2,5 \\
3 & Água & & & & 1,0 & 1,3 & 2,5 \\
4 & Cetonas & & & & 0,9 & 1,1 & 1,8 \\
5 & Ésteres & & & & & 0,53 & 2,0 \\
6 & Ácidos orgânicos & & & & & & 4,5 \\
\hline
\end{tabular}

Os parâmetros cruzados $(i \neq j)$ são calculados através das expressões mostradas no Quadro 3:

Quadro 3 - Regras de mistura da correlação de Hayden-O'Connell

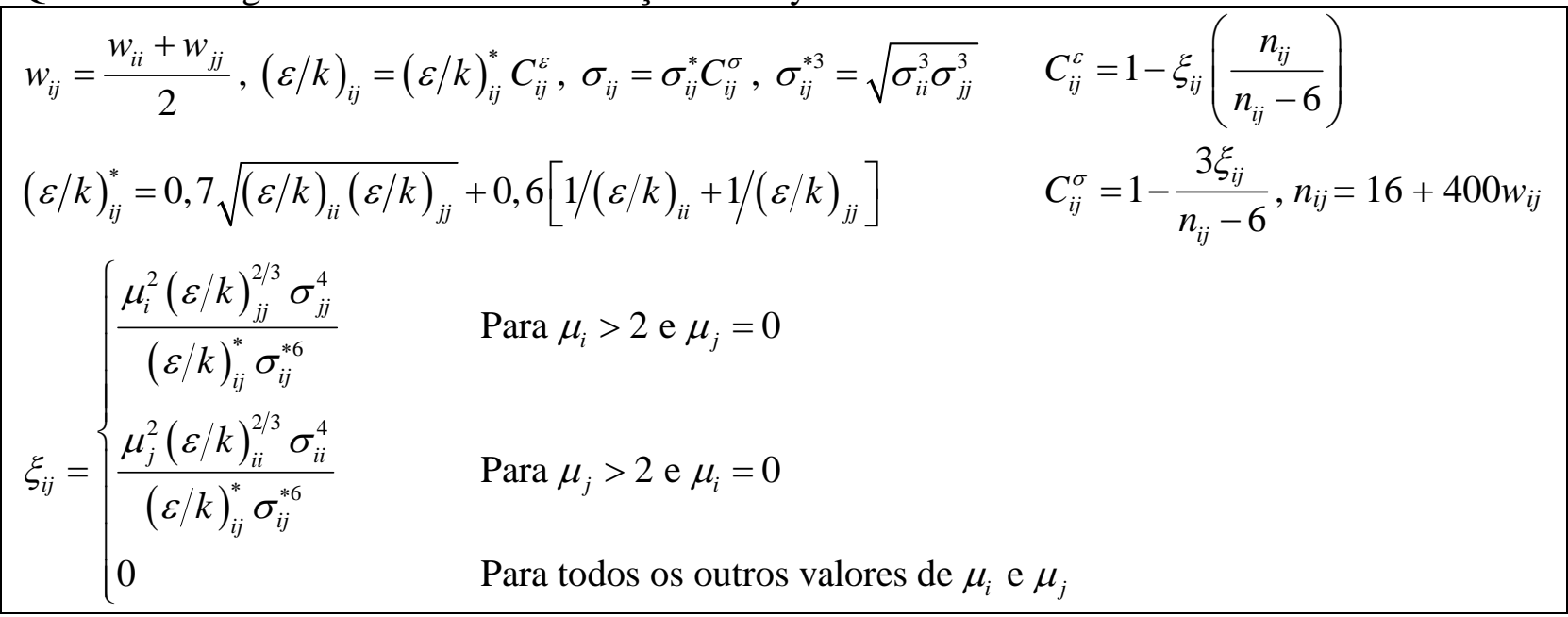


O procedimento computacional para estimativas das fugacidades das espécies químicas pode ser resumido através das seguintes etapas:

- Estimativas dos parâmetros $(\varepsilon / k)_{i i}, \sigma_{i i}$ e $w_{i i}$ e dos coeficientes do virial para as espécies puras presentes na mistura.

- Estimativas dos coeficientes do virial cruzados usando os parâmetros $(\varepsilon / k)_{i i}, \sigma_{i i}$ e $w_{i i}$

- Cálculo do segundo coeficiente do virial: $B=\sum_{i} \sum_{j} y_{i} y_{j} B_{i j}$.

- Cálculos dos coeficientes de fugacidades

Expansão na pressão: $\ln \hat{\phi}_{i}=\left(2 \sum_{j} y_{j} B_{i j}-B\right)\left(\frac{P}{R T}\right), \ln \phi=\sum_{i=1} y_{i} \ln \hat{\phi}_{i}$

Expansão na densidade molar: $\ln \hat{\phi}_{i}=\left(\frac{2}{V}\right) \sum_{j} y_{j} B_{i j}-\ln Z$ e $\ln \phi=\sum_{i=1} y_{i} \ln \hat{\phi}_{i}$

- Cálculos das fugacidades parciais: $\hat{f}_{i}=y_{i} \hat{\phi}_{i} P$

\section{METODOLOGIA}

A metodologia empregada para o desenvolvimento do aplicativo EXCEL/VBA é apresentada na Figura 1.

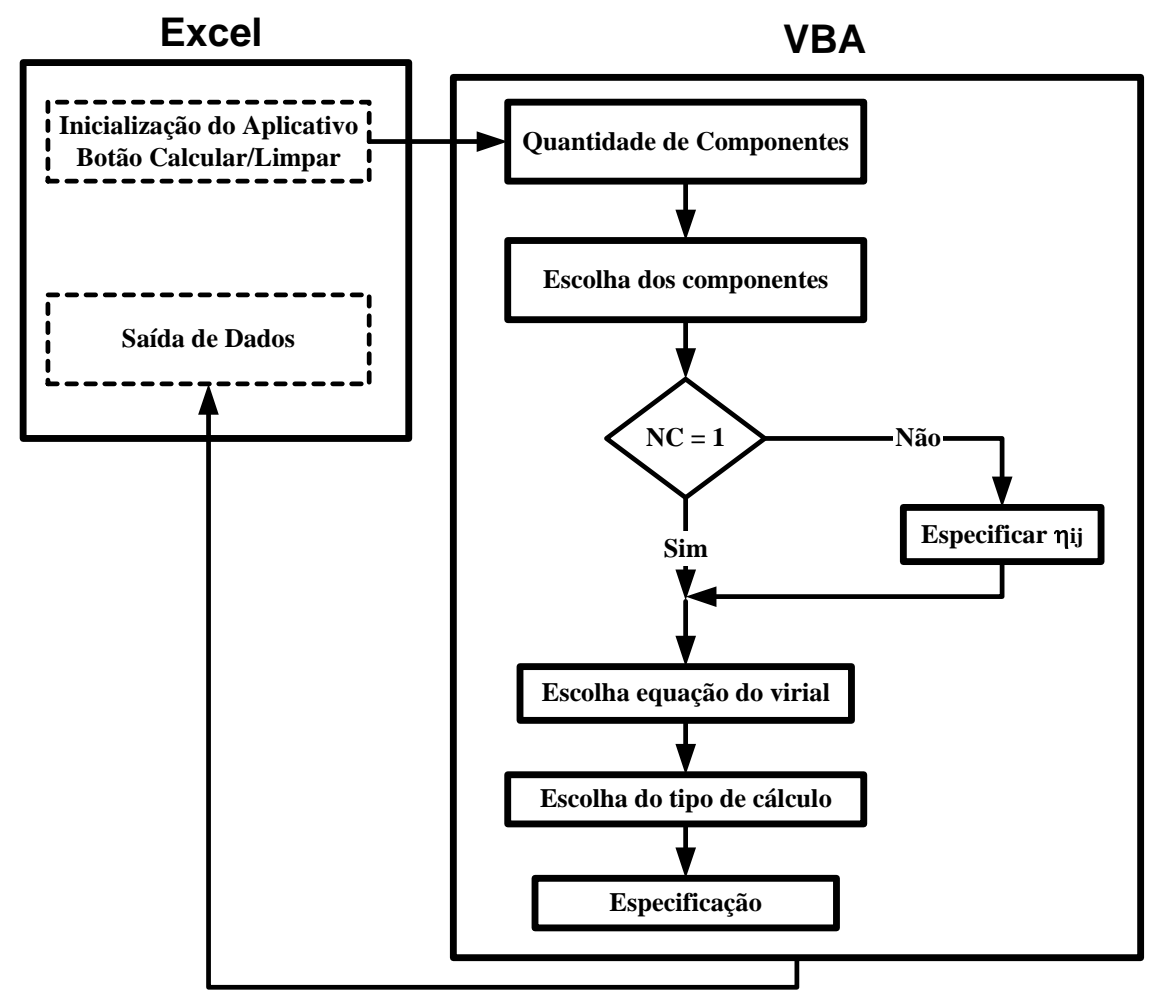

Figura 1 - Esquema do desenvolvimento do aplicativo 


\subsection{Etapas do desenvolvimento do aplicativo}

Para demonstrar as etapas do aplicativo criado, considere uma mistura de nitrogênio (1) - oxigênio (2) com y1 = 0,79 molar a 5 bar e $180 \mathrm{~K}$.

Banco de dados: $\mathrm{O}$ banco de dados contém as propriedades físicas de 60 espécies químicas (temperatura normal de ebulição, temperatura crítica, pressão crítica, volume molar crítico, fator, fator acêntrico e momento dipolo). Três conjuntos de propriedades físicas foram colocados no banco de dados: PF1 - reportadas por Reid et al. (1988); PF2 - reportadas por Poling et al. (2000) e PF3 - reportadas por Smith et al. (2007). Os raios médios de giração e os parâmetros de associação foram obtidos de Fredenslund et al. (1975), Prausntiz et al. (1980) e Sherwood et al. (1977).

Quantidade de espécies químicas: A caixa de diálogo para a escolha da quantidade de espécies presentes no sistema é mostrada na Figura 2. O usuário pode escolher na faixa de 1 a 12 espécies.

Escolha de espécies químicas: A caixa de diálogo para a escolha das espécies presentes é mostrada na Figura 3.

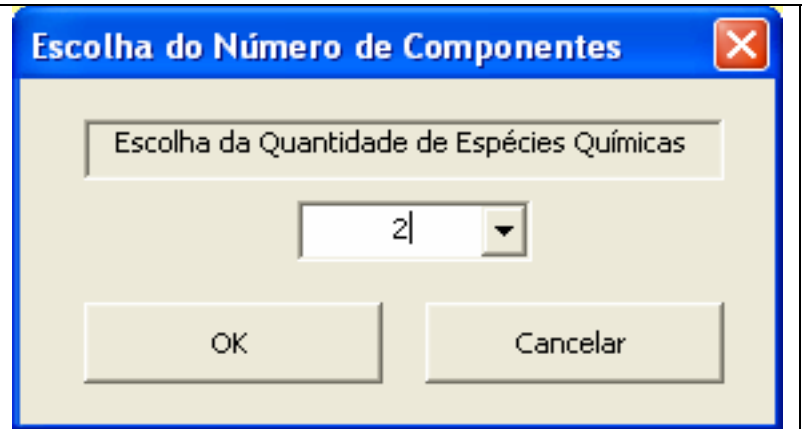

Figura 2 - Escolha da quantidade de espécies

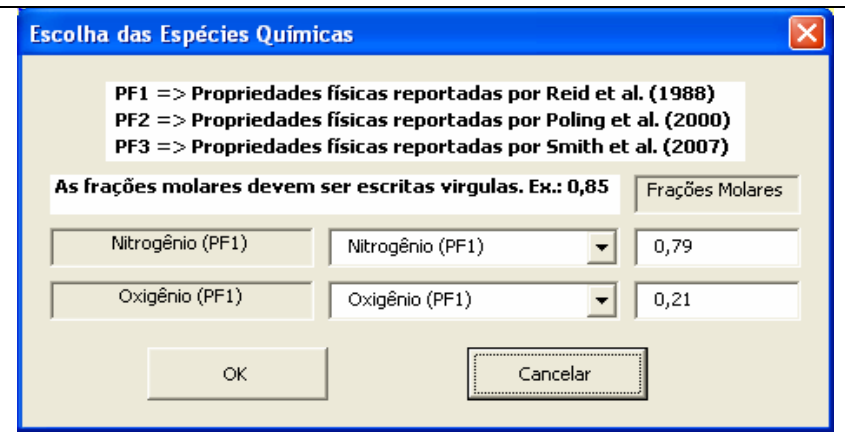

Figura 3 - Escolha das espécies Químicas

Especificações dos parâmetros de associação e solvatação: Se a escolha do número de espécies for maior que um, o aplicativo abre a caixa de diálogo para as especificações dos parâmetros de associação e solvatação (Figura 4).

Escolha da equação do virial: As opções para os cálculos das propriedades volumétricas e fugacidades são: equação do virial da expansão na pressão e expansão na densidade molar (Figura5).

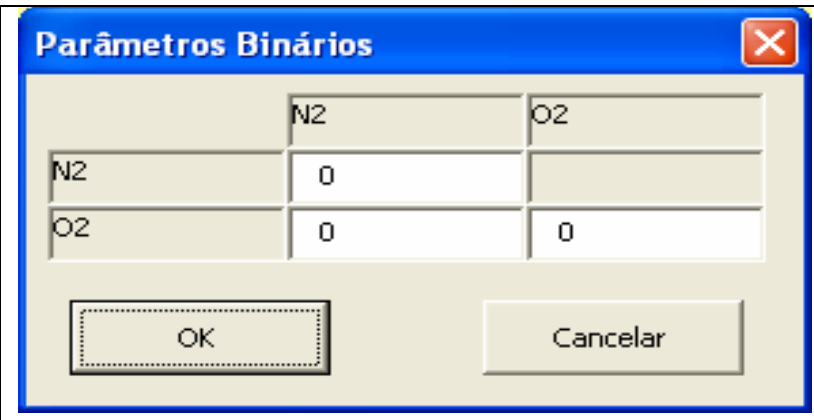

Figura 4- Especificações dos parâmetros

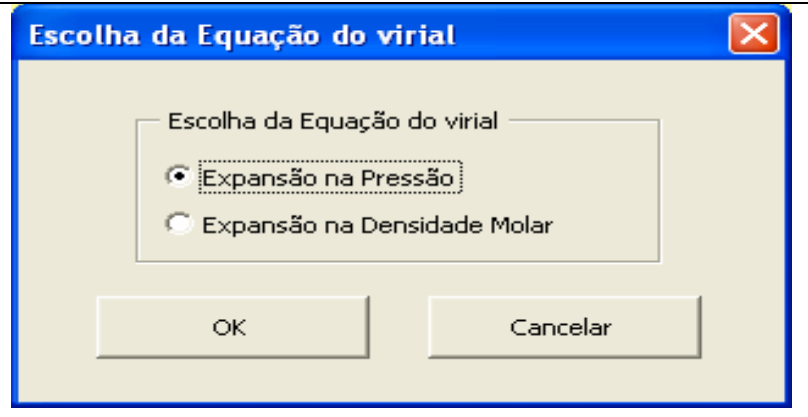

Figura 5 - Especificações dos parâmetros 
Escolha do tipo de variáveis independentes: A caixa de diálogo para a escolha das variáveis intensivas disponíveis para o cálculo é apresentada na Figura 6.

Especificações: A caixa de diálogo para as especificações da temperatura e da pressão é apresentada na Figura 7.

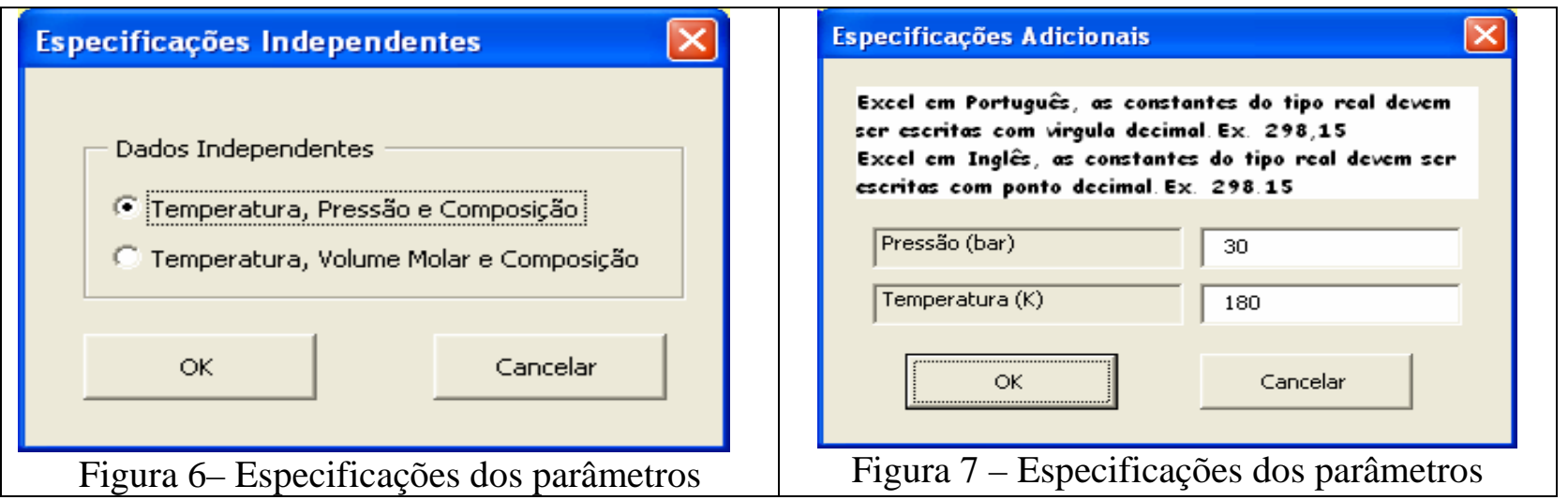

\section{RESULTADOS - VALIDAÇÃO DO APLICATIVO}

Vários sistemas foram utilizados para a validação do aplicativo proposto, ou seja, para a verificação das eficiências numéricas dos subprogramas codificados para as estimativas das fugacidades através da equação do virial. Este trabalho mostra apenas alguns resultados obtidos.

Coeficiente de fugacidade do n-butano: Na temperatura de 368,25 K, Fredenslund et al. (1975) reportam o valor experimental do segundo coeficiente do virial do n-butano igual a $-444,2 \mathrm{~cm}^{3} / \mathrm{mol}$. A comparação entre os valores reportados pelos pesquisadores e os estimados neste trabalho é apresentada na Tabela 3. As propriedades físicas utilizadas nas estimativas são: a) PF1: $T_{c}=425,2 \mathrm{~K}, P_{c}=38$ bar e $w=$ 0,199; b) PF2: $T_{c}=425,12 \mathrm{~K}, P_{c}=37,96$ bar e $w=0,2$ e c) PF3: Tc $=425,1 \mathrm{~K}, P_{c}=37,96$ bar e $w=0,2$. O segundo coeficiente do viral calculado é: a) PF1: $B=-436,54 \mathrm{~cm}^{3} / \mathrm{mol}$; b) PF2: $B=-436,74 \mathrm{~cm}^{3} / \mathrm{mol}^{\text {; }}$ c) PF3: $\mathrm{B}=-436,67 \mathrm{~cm}^{3} / \mathrm{mol}$

Tabela 3 - Resultados obtidos para o n-butano a 368,25 K

\begin{tabular}{lrccc|rccc}
\hline \multirow{2}{*}{$\mathrm{P}(\mathrm{atm})$} & \multicolumn{4}{c|}{ Expansão na pressão } & \multicolumn{4}{c}{ Expansão na densidade molar } \\
\cline { 2 - 9 } & Fred & PF1 & PF2 & PF3 & Fred & PF1 & PF2 & PF3 \\
\hline 1 & 0,9854 & 0,9857 & 0,9857 & 0,9857 & 0,9853 & 0,9856 & 0,9855 & 0,9855 \\
10 & 0,8632 & 0,8655 & 0,8654 & 0,8654 & 0,8514 & 0,8541 & 0,8540 & 0,8540 \\
\hline
\end{tabular}

a) Fred - valores reportados por Fredenslund et al. (1975) e b) PF1, PF2 e PF3 - são os valores calculados usando as propriedades físicas do banco de dados PF1, PF2 e PF3

Os resultados obtidos com os três conjuntos de propriedades físicas apresentam boas concordâncias quando comparados aos valores reportados por Fredenslund et al. (1975), tanto para estimativas usando a equação do virial na expansão da pressão como para a expansão na densidade molar. Pode se observado que as estimativas do segundo coeficiente do virial usando a correlação de Hayden-O'Connell com os três conjuntos de temperatura estão dentro das incertezas experimentais. 
Sistema nitrogênio (1) - metano (2): A comparação entre os resultados reportados por Smith et al. (2007) com os obtidos através do aplicativo para a mistura de $40 \%$ em base molar do nitrogênio (1) e metano (2) a $200 \mathrm{~K}$ e 30 bar é apresentada na Tabela 4. As propriedades físicas são: $T_{c 1}=33,2 \mathrm{~K}, T_{c 2}=$ 369,8 K, $P_{c 1}=13$ bar e $P_{c 2}=42,5$ bar. Os raios médios de giração são: $\bar{R}_{1}=0,547 \AA$ e $\bar{R}_{2}=1,118 \AA$. Os parâmetros de associação e solvatação são: $\eta_{i j}=0$ para $i$ e $j=1,2$.

Tabela 4 - Resultados finais para a mistura de nitrogênio (1) - metano (2) a $200 \mathrm{~K}$

\begin{tabular}{lccccccc}
\hline & $\mathrm{B}_{11}$ & $\mathrm{~B}_{22}$ & $\mathrm{~B}_{12}$ & $\mathrm{~B}$ & $\mathrm{Z}$ & $\hat{\phi}_{1}$ & $\hat{\phi}_{2}$ \\
\hline Smith & $-35,2$ & $-105,0$ & $-59,8$ & $-72,14$ & 0,870 & 0,9511 & 0,8324 \\
PF1 & $-40,79$ & $-107,29$ & $-66,70$ & $-77,17$ & 0,861 & 0,9380 & 0,8275 \\
PF2 & $-40,70$ & $-107,58$ & $-66,71$ & $-77,26$ & 0,861 & 0,9382 & 0,8271 \\
PF3 & $-40,67$ & $-107,65$ & $-66,71$ & $-77,28$ & 0,861 & 0,9383 & 0,8270 \\
\hline
\end{tabular}

$B$ é o segundo coeficiente do virial da mistura; $B_{i j}$ são os coeficientes do virial das espécies puras e cruzadas; $Z$ é o fator de compressibilidade e $\hat{\phi}_{i}$ é coeficiente de fugacidade da espécie $i$.

Os resultados mostrados na Tabela 4 indicam que os valores estimados através do aplicativo proposto apresentam boas concordâncias com os valores reportados por Smith et al. (2007).

Sistema ternário metano (1) - etano (2) - propano (3): A comparação entre os resultados reportados por Smith et al. (2007) com os obtidos através do aplicativo para a mistura de $21 \%$ em base molar do metano (1), 43 \% de etano (2) e $36 \%$ de propano (3) a 373,15 K e 35 bar é apresentada na Tabela 5 . As propriedades físicas são: a) PF1: $T_{c 1}=190,4 \mathrm{~K}, T_{c 2}=305,4 \mathrm{~K}, T_{c 3}=369,8 \mathrm{~K}, P_{c 1}=46 \mathrm{bar}, P_{c 2}=48,8 \mathrm{bar}$, $P_{c 3}=42,5$ bar; b) PF2: $T_{c 1}=190,56 \mathrm{~K}, T_{c 2}=305,32 \mathrm{~K}, T_{c 3}=369,83 \mathrm{~K}, P_{c 1}=45,99$ bar, $P_{c 2}=48,72$ bar, $P_{c 2}$ $=42,48$ bar; c) PF3: $T_{c 1}=190,6 \mathrm{~K}, T_{c 2}=305,3 \mathrm{~K}, T_{c 3}=369,8 \mathrm{~K}, P_{c 1}=45,99 \mathrm{bar}, P_{c 2}=48,72 \mathrm{bar}, P_{c 3}=$ 42,48 bar. Os raios médios de giração são: $\bar{R}_{1}=1,118 \AA$ А $, \bar{R}_{2}=1,826 \AA$ e $\bar{R}_{3}=2,431 \AA$. Os parâmetros de associação e solvatação são: $\eta_{i j}=0$ para $i$ e $j=1,2$, 3 .

Tabela 5 - Resultados finais para a mistura de nitrogênio (1) - metano (2) a $200 \mathrm{~K}$

\begin{tabular}{lcccccc}
\hline & $\hat{\phi}_{1}$ & $\hat{\phi}_{2}$ & $\hat{\phi}_{3}$ & $\hat{f}_{1}$ (bar) & $\hat{f}_{2}$ (bar) & $\hat{f}_{3}$ (bar) \\
\hline Smith & 1,0190 & 0,8810 & 0,7750 & 7,491 & 13,254 & 9,764 \\
PF1 & 1,0209 & 0,8784 & 0,7792 & 7,504 & 13,220 & 9,817 \\
PF2 & 1,0208 & 0,8783 & 0,7790 & 7,503 & 13,219 & 9,815 \\
PF3 & 1,0207 & 0,8784 & 0,7790 & 7,502 & 13,219 & 9,816 \\
\hline
\end{tabular}

Os resultados dos coeficientes de fugacidades e das fugacidades dos componentes presentes na misturas, mostrados na Tabela 5, indicam que os valores estimados através do aplicativo proposto apresentam excelentes concordâncias com os valores reportados por Smith et al. (2007). 


\section{CONCLUSÃO}

Este trabalho apresenta uma planilha capaz de fornecer com confiabilidade e rapidez as estimativas das propriedades volumétricas, de fugacidades de espécies puras, de mistura e de componentes de uma mistura. Deve ser observado que a precisão numérica dessas estimativas está associada à confiabilidade da equação de estado do virial truncada no segundo termo, que fornece boas estimativas das propriedades volumétricas para sistemas cuja densidade molar é inferior a 1/3 da densidade crítica, ou seja, em pressões baixas ou moderadas.

\section{REFERÊNCIA}

COSTA, N. A. Modelagem Matemática da Termodinâmica Química - Equações de Estado. Volume I. Documento pessoal, 2014a.

COSTA, N. A. Modelagem Matemática da Termodinâmica Química - Fugacidade/Coeficiente de Fugacidade. Volume IV. Documento pessoal, 2014b.

FREDENSLUND, A. GMEHLING, J; RASMUSSEN, P. Vapor-Liquid Equilibria Using UNIFAC - A Group-Contribution Method. Elsevier Scientific Publishing Company, 1977.

HAYDEN, J. G; O’CONNELL, J. P. A Generalized Method for Predicting Second Virial Coefficient. Ind. Eng. Chem. Proc. Des. Dev. Vol. 14, 209-216.

REID, R. C; PRAUSNITZ, J. M; POLING, B. E. The Properties of Gases \& Liquids. Fourth Edition. McGraw-Hill Book Company, 1988.

POLING, B. E; PRAUSNITZ, J. M; O'CONNEL, J. P. The Properties of Gases and Liquids. Fifth Edition. McGraw-Hill, 2000.

PRAUSNITZ, J. M; ANDERSON, T. F; GRENS, E. A; ECKERT, C. A; HSIEH, R; O'CONNELL, J. P. Computer Calculations for Multicomponent Vapor-Liquid and Liquid-Liquid Equilibria. Prentice-Hall, Inc, 1980.

SHERWOOD, T. K., REID, R. C; PRAUSNITZ, J. M. The Properties of Gases \& Liquids. Third Edition. McGraw-Hill Book Company, 1977.

SMITH, J. M; Van Ness, H. C; ABBOTT, M. M. Introdução à Termodinâmica da Engenharia Química. Quinta Edição. LTC; 2007. 